\title{
Haemodynamic and oxygen transport response during exchange transfusion for severe falciparum malaria
}

\author{
S.C. Beards, G.M. Joynt and J. Lipman
}

Baragwanath Intensive Care Unit and Department of Anaesthesia, University of Witwatersrand, PO Box 2013, Bertsham, Soweto, South Africa

\begin{abstract}
Summary: We describe the haemodynamic and oxygen transport response in a patient undergoing exchange transfusion for severe falciparum malaria. We found that exchange transfusion produced a significant increase in left ventricular stroke work index, systemic oxygen delivery and oxygen consumption. This potentially beneficial effect of exchange transfusion has not been reported previously.
\end{abstract}

\section{Introduction}

In recent years there has been a marked increase in the incidence of malaria due to Plasmodium falciparum in both developed countries and the Third World. ${ }^{1,2}$ Despite improvements in intensive care management and schizonticidal drug therapy the mortality remains high. ${ }^{2}$ The use of exchange transfusion as an adjunct to conventional treatment has been said to be of benefit in severe or complicated cases, ${ }^{1,3-8}$ although controlled trials have never established its use. No information exists on the influence of exchange transfusion on systemic haemodynamics of global oxygen transport.

\section{Case history}

A 30 year old black patient presented with a 2-day history of generalized muscular weakness, fever, rigors and vomiting. His heart rate was 130 beats/ minute, blood pressure $120 / 70 \mathrm{mmHg}$, respiratory rate $38 /$ minute, and temperature $39^{\circ} \mathrm{C}$. $\mathrm{He}$ had recently returned from a holiday in Mozambique and had taken malaria chemoprophylaxis. On examination the relevant positive findings were that he was jaundiced, confused and was passing dark-coloured urine. The lung fields were clear on auscultation and the tip of his spleen palpable. Examination of a thin blood film for malaria revealed a $10 \%$ parasitaemia with ring form trophozoites of $P$. falciparum. Haematological investigations revealed a platelet count of $25 \times 10^{9} / 1$ and haemoglobin $10.7 \mathrm{~g} / \mathrm{dl}$. Serum sodium was $129 \mathrm{mmol} / \mathrm{l}$, potassium $4.2 \mathrm{mmol} / \mathrm{l}$,

Correspondence: S.C. Beards, M.B., Ch.B., F.R.C.A., 24 Greenwood Drive, Wilmslow, Cheshire SK9 2RW, UK. Accepted: 8 June 1994 urea $24.4 \mathrm{mmol} / \mathrm{l}$ and creatinine $242 \mu \mathrm{mol} / \mathrm{l}$. Indirect and direct levels of bilirubin were elevated to $96 \mu \mathrm{mol} / 1$ and $48 \mu \mathrm{mol} / 1$, respectively. Arterial blood gases on room air revealed a $\mathrm{PaO}_{2}$ of $8.8 \mathrm{kPa}$, $\mathrm{PaCO}_{2} 3.7 \mathrm{kPa}, \mathrm{pH} 7.45$, bicarbonate $19.7 \mathrm{mmHg}$ and base excess $-2.4 \mathrm{mmol} / \mathrm{l}$.

Within 4 hours of admission, the level of parasitaemia had increased to $15 \%$ and the patient's conscious level had deteriorated (Glasgow coma score 11/15). The patient's calculated Apache II score was 21 . He was given an initial loading dose of $20 \mathrm{mg} / \mathrm{kg}$ of intravenous quinine and commenced on $10 \mathrm{mg} / \mathrm{kg} 8$ hourly thereafter. Systemic and pulmonary arterial catheters were inserted and a large double-bore catheter (11.5 French gauge, Quinton Instrument Co., Seattle, WA) inserted into the femoral vein as per unit policy. Blood was removed in $500 \mathrm{ml}$ aliquots from one port of the dialysis catheter using a $50 \mathrm{ml}$ syringe. A roller pump was attached to the other port of the catheter to enable rapid infusion of fluid. Three litres of blood were removed overall and were replaced with 3 litres of fresh whole blood and 0.5 litres of colloid (Haemacell, Hoechst) as shown in Figure 1. The entire procedure was performed within 3 hours. No inotropic support was required throughout the exchange. Full haemodynamic and oxygen transport measurements were performed at each phase of the exchange transfusion. Cardiac output was measured by the thermodilution technique (Nikon Koden, Lifescope 12 series, Japan). The mean of three measurements, each using $10 \mathrm{ml}$ of ice-cold $5 \%$ dextrose, was calculated. Arterial and mixed venous oxygen contents were obtained from a co-oximeter (Lexington IL482) and gas analysis performed (ABL 500 machine, Radiometer, Copenhagen).

The patient's conscious level improved rapidly during the procedure (Glasgow coma score 15/15), 
he was discharged from the intensive care unit after 3 days and made an uneventful recovery.

\section{Haemodynamic and oxygen transport changes}

The haemodynamic and oxygen transport variables were assessed by regression coefficient analysis using four independent variables. ${ }^{7}$ Comparison was made with the fluid balance at each step of the exchange (Figure 1), with the cumulative total exchanged and with the haemoglobin at each step (Figure 2).

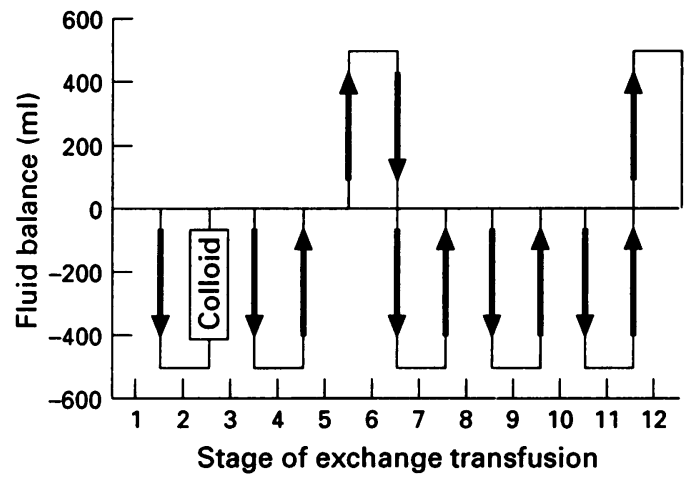

Figure 1 A demonstration of the fluid shifts of the 12 stages of the exchange transfusion. Each arrow represents one stage of the exchange. At each stage blood was either administered or withdrawn in the volumes indicated on the vertical axis. Colloid (Haemacell) was given during stage 2 only.
Regression analysis using the fluid balance as the $\frac{2}{3}$

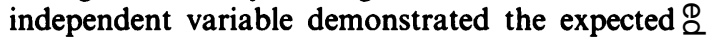
changes in central venous pressure (CVP) $\subseteq$ $(P<0.001)$ and pulmonary capillary wedge pres- $\vec{F}$ sure (PCWP) $(P<0.001)$ (Table I). Changes in $\stackrel{\oplus}{9}$ systolic (PASP) $(P<0.001)$, diastolic (PADP) $(P<0.005)$ and mean (MPAP) $(P<0.001)$ pulmonary artery pressures and in right ventricular $\frac{\infty}{\sigma}$ stroke work index $(\mathrm{RVSWI})(P<0.05)$ also closely $\varrho$ mirrored changes in fluid load (Table I and Figure ${ }^{\circ}$ $3)$. In the systemic circulation, changes in mean $\vec{O}$ arterial pressure (MAP) $(P<0.001)$ and arterial diastolic pressure (DBP) $(P<0.001)$ were also $\vec{\omega}$ related to changes in fluid load, although arterial systolic pressure (SBP) was not. None of these

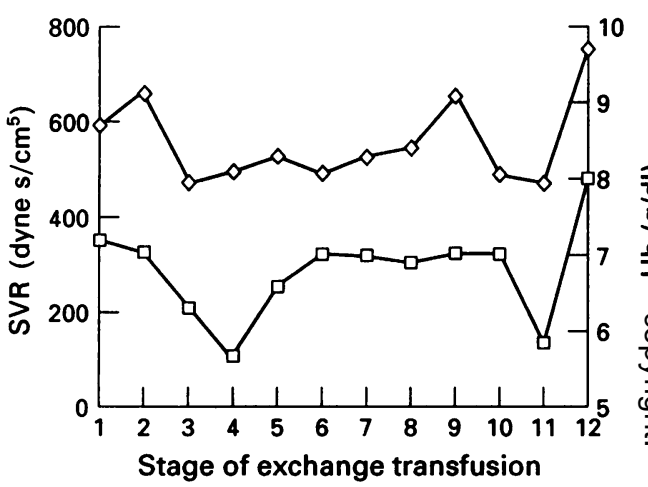

Figure 2 The changes in systemic vascular resistance (SVR, $\square)$ and haemoglobin $\mathrm{Hb}(\diamond)$ during each stage of the exchange transfusion.

Table I Changes in haemodynamic variables during exchange transfusion. The column designated 'Volume exchanged' refers to the total cumulative fluid balance in millilitres as the exchange tranfusion progresses

\begin{tabular}{lcccccccc}
\hline $\begin{array}{l}\text { Volume } \\
\text { exchanged }\end{array}$ & $M A P$ & $D B P$ & $C V P$ & $P C W P$ & $C I$ & $L S W I$ & $R S W I$ & $S V R$ \\
\hline 0 & 72 & 52 & 12 & 18 & 4.42 & 30 & 6.28 & 586 \\
-500 & 71 & 55 & 7 & 12 & 4.14 & 33 & 9.13 & 668 \\
0 & 79 & 59 & 13 & 20 & 5.90 & 44 & 12.90 & 483 \\
-500 & 70 & 50 & 7 & 13 & 5.44 & 38 & 10.20 & 500 \\
0 & 82 & 60 & 13 & 23 & 5.60 & 41 & 13.40 & 532 \\
500 & 85 & 67 & 16 & 25 & 5.98 & 44 & 12.70 & 498 \\
-500 & 67 & 50 & 4 & 9 & 5.16 & 37 & 8.30 & 527 \\
0 & 80 & 62 & 12 & 18 & 5.36 & 42 & 12.20 & 548 \\
-500 & 75 & 57 & 5 & 8 & 4.60 & 37 & 7.24 & 657 \\
0 & 82 & 60 & 11 & 17 & 6.23 & 53 & 15.50 & 492 \\
-500 & 72 & 51 & 5 & 9 & 6.09 & 47 & 11.40 & 475 \\
500 & 92 & 71 & 10 & 17 & 4.67 & 44 & 14.30 & 759 \\
\hline
\end{tabular}

MAP $=$ mean arterial pressure $(\mathrm{mmHg}) ; \mathrm{DBP}=$ diastolic blood pressure $(\mathrm{mmHg}) ; \mathrm{CVP}=$ central venous pressure $(\mathrm{mmHg}) ; \mathbf{P C W P}=$ pulmonary capillary wedge pressure $(\mathrm{mmHg}) ; \mathrm{CL}=\mathrm{car}$ diac index $\left(1 /\right.$ minute $\left./ \mathbf{M}^{2}\right)$; LSWI = left ventricular stroke work index $\left(\mathrm{gM} / \mathrm{M}^{2}\right)$; RSWI = right ventricular stroke work index $\left(\mathrm{gM} / \mathrm{M}^{2}\right) ; \mathrm{SVR}=$ systemic vascular resistance $\left(\right.$ dyne seconds $\left./ \mathrm{cm}^{5}\right)$. 


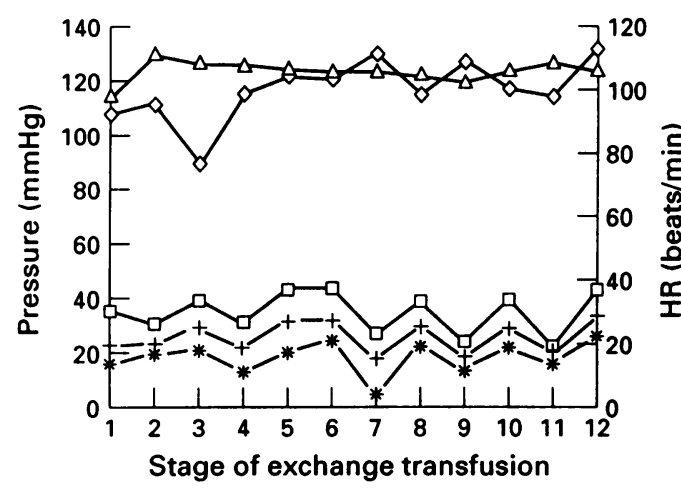

Figure 3 The stable heart rate $(H R, \Delta)$ systolic arterial pressure $(\diamond)$, and changes in the pulmonary artery systolic $(\square)$, diastolic $\left({ }^{*}\right)$ and mean $(+)$ pressures at each stage of the exchange transfusion.

variables showed any significant relationship with the cumulative exchange volume or with changes in haemoglobin.

Regression analysis using the cumulative volume of the exchange transfusion at each step demonstrated a significant increase in left ventricular stroke work index (LVSWI) $(P<0.02)$ and increases in both oxygen delivery index $\left(\mathrm{DO}_{2}\right)$ $(P<0.03)$ and oxygen consumption index $\left(\mathrm{VO}_{2}\right)$ $(P<0.03)$ (Figure 4).

$\mathrm{VO}_{2}$ was directly proportional to $\mathrm{DO}_{2}$ throughout the observed range (Figure 4$)(P<0.001)$. Changes in cardiac index (CI) were minimal throughout the exchange and showed no significant correlation with fluid balace, cumulative exchange volume or haemoglobin.

The level of haemoglobin showed significant fluctuation during the exchange due to the infusion of colloid solution (Haemacell) during the initial transfusion. The variation in haemoglobin was significantly related to changes in the systemic vascular resistance (SVR) $(P<0.01)$ and DBP $(P<0.05)$ but was independent of all other variables (Figure 2). Variations in systemic vascular resistance (SVR) were also independent of other measured variables.

\section{Discussion}

The use of exchange transfusion for the treatment of cerebral malaria was first described by Gyr et al. in $1974^{6}$ and has since gained increasing acceptance as an adjunct to conventional drug therapy in severe or complicated cases. Although all previous reports of exchange transfusion have been essentially favourable, the indications for use, mechanism of action and optimal method of exchange remain contentious.

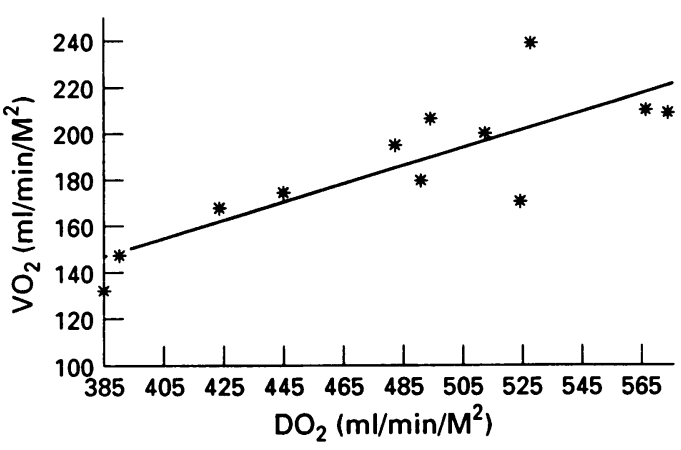

Figure 4 A graph demonstrating the supply dependency relationship of the oxygen delivery index $\left(\mathrm{DO}_{2}\right)$ and oxygen consumption index $\left(\mathrm{VO}_{2}\right)$ during exchange transfusion.

The actual mechanisms of organ damage in malaria remain unknown. Infected erythrocytes have significant membrane deformities and demonstrate increased rigidity, which is proportional to the maturity of the parasite. These effects combine to produce deleterious changes in rheology and, in postmortem cases, small vessels are congested with infected erythrocytes containing mature trophozoites and schizonts. ${ }^{2,8}$ Infected cells also demonstrate cytoadherence to a range of cell membrane glycoproteins that are expressed by capillary and venular epithelium leading to sequestration of infected erythrocytes in small vessels. ${ }^{2}$ These changes in erythrocyte behaviour gave rise to the 'mechanical theory' of organ damage, which attempts to explain the pathophysiological changes in severe malaria on the basis of small vessel obstruction and secondary ischaemia. Despite previous widespread acceptance, the mechanical theory is no longer believed to provide an adequate explanation of the pathological changes that occur in severe cases. In a study of adult patients with cerebral malaria, there was no demonstrable decrease in cerebral blood flow as would be predicted by the mechanical hypothesis, although cerebral oxygen extraction was significantly reduced and cerebral lactate production increased. ${ }^{9}$

Rapid reduction of parasitaemia with an associated improvement in rheology, haemodynamics and oxygen transport has been suggested as a mechanism for the rapid improvement in neurological status, which may occur during exchange as in the present case. ${ }^{1} \mathrm{~A}$ reduction in circulating cytokines and parasitic toxins has also been suggested as a possible mechanism for this dramatic response, ${ }^{\prime}$ In the present case a gradual increase in oxygen delivery occurred during the transfusion and was significantly related to the cumulative volume of the exchange transfusion 
and to the consequent reduction in parasitaemia. A simultaneous increase in peripheral oxygen consumption was also demonstrated and was shown to be dependent on the increase in oxygen delivery. Although the possibility of mathematical coupling exists, this would not account for the improvement in the patient's level of consciousness.

It is of interest that the study of Warrell et al. ${ }^{9}$ demonstrated that cerebral blood flow in patients with cerebral malaria is low in relation to arterial oxygen content, suggesting a loss of the normal regulatory response. In the presence of this decrease of cerebral oxygen delivery, increases in systemic $\mathrm{DO}_{2}$ as demonstrated in the current study could lead to a rapid and significant improvement in cerebral oxygenation.

The changes in SVR in the present case correlated with changes in haemoglobin and showed no relationship with the level of parasitaemia or the cumulative exchange volume. This observation is not in keeping with the mechanical hypothesis that increased peripheral oxygen delivery results from an improvement in blood rheology with a consequent decrease in vascular resistance due to decreasing parasitaemia.

In the present case, reactive haemodynamic changes in response to changes in fluid load were

\section{References}

1. Phillips, P., Nantel, S. \& Benny, B. Exchange transfusion as an adjunct to the treatment of severe falciparum malaria: case report and review. Rev Inf Dis 1990, 12: 1100-1108.

2. World Health Organisation Division of Control of Tropical Diseases. Severe and complicated malaria. Trans $R$ Soc Trop Med Hyg 1990, 84: 1-65.

3. White, N.J., Warrell, D.A., Looareesuwan, S. et al. Pathophysiological and prognostic significance of CSF lactate in cerebral malaria. Lancet 1985, i: 776-778.

4. Salord, F., Allaouchiche, B., Gaussorgues, P. et al. Severe falciparum malaria (21 cases). Intensive Care Med 1991, 17: 449-454.

5. Looareesuwan, S., Phillips, R.E., Karbwang, J. et al. Plasmodium falciparum hyperparasitaemia: use of exchange transfusion in seven patients and a review of the literature. $Q \mathrm{~J} \mathrm{Med}$ 1990, 75: $471-481$. entirely transient and insufficient to be of clinical significance. Cardiac output, cardiac index and $\stackrel{\mathbb{Q}}{\complement}$ systolic arterial pressure were maintained through- $c$ out the exchange and oxygen delivery did not fall $\Rightarrow$ significantly below its initial value at any stage. An $\stackrel{\rho}{+}$ increase in LVSWI was the only sustained? haemodynamic change in response to transfusion, $\frac{}{2}$ the mechanism underlying this remains unclear, $\frac{N}{\vec{D}}$ although it may reflect improved oxygen delivery $\triangle$ to the myocardium.

The findings in the present case demonstrate that $\vec{\circ}$ partial volume exchange transfusion is an effective and haemodynamically well-tolerated method of $\vec{\omega}$ reducing parasitaemia in severe falciparum $\stackrel{\odot}{\circ}$ malaria. Exchange transfusion is associated witho an increase in oxygen delivery and oxygen con- 3 . sumption, which may partly explain the rapid $\partial$ improvements in conscious level which can occur in patients with cerebral malaria during exchange 0 transfusion.

\section{Acknowledgement}

We are grateful to Dr J.D. Edwards for his help and advice in the preparation of this manuscript.

6. Gyr, K., Speck, B., Ritz, R. et al. Zerebrale malaria tropica mit schwarzwasserfieber. Ein actualles diagnostisches und therapeutisches problem. Schweiz Med Wochensch 1974, 104: $1628-1630$.

7. Altman, D.G. Practical Statistics for Medical Research. Chapman and Hall, London, 1991, pp. 29-46.

8. Ahlqvist, J. Decreased red cell deformability and vascular obstruction in falciparum malaria illustrated by a fatal case. Scand J Haematol 1985, 35: 531-535.

9. Warrell, D.A., Veall, N., Chanthavanich, P. et al. Cerebra anaerobic glycolysis and reduced oxygen transport in human cerebral malaria. Lancet 1988 , i: 534-538. 